\title{
Evaluation of two commercially available methods used for the rapid detection of ESBL-producing strains
}

\author{
Concetta De Luca', Erminia AM Casari', Elisabetta Nucleo², Antonella Ferrario', Roberta \\ Migliavacca², Laura Pagani² \\ I Laboratorio Analisi, Sezione di Microbiologia, IRCCS Istituto Clinico Humanitas, Rozzano (MI) \\ 2 Dipartimento SMEC, Sezione di Microbiologia, Università di Pavia
}

Key words: ESBL, Detection, Methods

Valutazione di due metodi commerciali per la rilevazione rapida di ceppi produttori di ESBL

\section{SUMMARY}

Detection of ESBL production by Enterobacteriaceae remains a challenge for microbiologists. Although recent changes in the breakpoints of third-generation cephalosporins decreased the likelihood of reporting ESBL producers as susceptible to these compounds, ESBL detection is of interest for prevention of dissemination of ESBL-producers by cross-transmission and for epidemiological purpose. The aim of this study was to evaluate the sensitivity and the specificity of two commercial methods suitable for rapid ESBL-detection in Gram negative bacilli: the ChromID ESBL medium (bioMérieux) and the Cica-ß-Test (Mast Group, Merseyde, UK).

121 Enterobacteriaceae collected between February 2008 and April 2009 at the Laboratory Analysis IRCCS Humanitas were tested for ESBL-production by Phoenix ${ }^{T M}$, E-test (ESBL reference test), ChromID ESBL medium and Cica- $\beta$-Test.

ChromID showed $100 \%$ of sensitivity and specificity for the screening of ESBL in E. coli; lower values of specificity were found in the case of P. mirabilis (81\%) and Klebsiella spp. (92\%).

The Cica- $\beta$-Test always showed high specificity levels, but the poor sensitivity found for both $E$. coli $(90 \%)$, P. mirabilis (73\%) and Klebsiella spp. (85\%), discourages its use for screening of ESBL in Gram negative bacilli from blood-cultures. Rapid identification of ESBL producers is of interest to implement hygiene precautions. In that case, using a very sensitive primary test is of major interest.

\section{INTRODUZIONE}

La crescente diffusione di microrganismi ESBLproduttori rappresenta un problema sia in ambito nosocomiale che comunitario (15). Le scelte terapeutiche, nel caso di infezioni gravi provocate da tali batteri, risultano limitate ed il rischio di mortalità elevato $(6)$.

Le ESBL possono conferire resistenza ai monobattami, alle cefalosporine a spettro esteso come cefotaxime, ceftriaxone e ceftazidime ed, in alcuni casi, anche a cefepime e cefpirome (1).

I geni che codificano per le ESBL sono generalmente localizzati a livello plasmidico e vengono acquisiti mediante trasferimento orizzontale.

oirel, et al (13) hanno dimostrato un'associazione tra la produzione di ESBL e l'espressione di resistenza qnrA-mediata ai fluorochinoloni. Ciò è attribuibile alla frequente co-presenza, a livello plasmidico, di geni qnr e di geni codificanti per vari tipi di ESBL, AmpC (5) o metallo- $\beta$-lattamasi (10).

Originatisi da mutazioni degli enzimi progenitori TEM e SHV, ad oggi sono stati descritti numerosi tipi di ESBL, quali le CTX-M, divenute predominanti nell'ultimo decennio (8).
Il rilievo della produzione di ESBL negli enterobatteri rimane una sfida per i microbiologi. Sebbene recenti cambiamenti nei breakpoint delle cefalosporine di terza generazione abbiano diminuito la possibilità di refertare i microrganismi ESBL-produttori come sensibili a queste molecole (CLSI Giugno 2010, EUCAST Version 1.1 April 2010), l'identificazione delle ESBL risulta fondamentale al fine sia di prevenire la disseminazione mediantre cross-trasmissione degli ESBLproduttori sia a scopo epidemiologico.

Uno studio condotto nel 2008 (17) ha inoltre chiaramente dimostrato l'importanza della rapidità nell'impostazione di una terapia mirata, in caso di infezione causata da batteri ESBL-produttori.

Risulta pertanto indispensabile disporre di metodi diagnostici rapidi, sensibili e che non necessitino di ulteriori test di conferma nel permettere la corretta identificazione dei microrganismi produttori di tali enzimi idrolizzanti.

Diversi sono i test utilizzabili allo scopo di rilevare i batteri ESBL-produttori. La maggior parte di questi sfrutta due caratteristiche fondamentali e distintive dei batteri ESBL-produttori: la ridotta 
sensibilità alle ossimino-cefalosporine ed all'aztreonam e la restituzione di sensibilità a tali molecole in presenza di acido clavulanico (o di altri inibitori quali tazobactam e sulbactam).

Per la conferma fenotipica della produzione di ESBL, le Linee guida CLSI 2009 raccomandavano l'uso di test allestiti utilizzando un inibitore delle $\beta$-lattamasi in presenza di antibiotici indicatori quali ceftazidime, cefotaxime, aztreonam e ceftriaxone (2).

I test di laboratorio classicamente utilizzati per lo screening ed il rilievo di stipiti batterici produttori di ESBL comprendono il test del doppio disco e l'Etest (10), oppure si avvalgono degli algoritmi identificativi dei sistemi automatizzati "esperti" (18).

Nel test del doppio disco, eseguito su terreno agarizzato Mueller Hinton, quattro dischetti di carta bibula imbevuti di cefotaxime, ceftazidime, cefepime ed aztreonam vengono posti ad una distanza di $25 \mathrm{~mm}$ centro a centro da un dischetto contenente amoxicillina + acido clavulanico.

La produzione di ESBL viene evidenziata mediante la visualizzazione della presenza di caratteristiche distorsioni dell'alone di inibizione indicanti azione sinergica tra l'inibitore delle ESBL e gli antibiotici posti esternamente.

L'Etest, sviluppato da AB BioDisk (bioMérieux, Marcy-l'Etoile, France) si basa, per la rilevazione della produzione di ESBL, sullo sviluppo di effetto sinergico tra cefotaxime, ceftazidime ed acido clavulanico; tale metodo e si è rivelato, per sensibilità e facilità di esecuzione, di grande utilità a livello diagnostico (11).

Recentemente sono state sviluppate da AB BioDisk strip contenenti cefepime-clavulanato utili per la rilevazione degli enzimi di tipo AmpC (11).

In uno studio condotto nel 2008 su 114 stipiti di Enterobacteriaceae, è stata valutata la capacità di rilevare la produzione di enzimi di tipo ESBL da parte dei sistemi automatizzati Phoenix ${ }^{\mathrm{TM}}$ (BD Diagnostic Systems, Sparks, MD) e Vitek 2 (bioMérieux, Marcy-l'Etoile, France) (4).

Per lo screening della produzione di ESBL sono stati inoltre recentemente proposti alcuni terreni selettivi come Mac Conkey agar addizionati di concentrazioni differenti di cefotaxime o ceftazidime (12).

Scopo di questo lavoro è stato valutare sensibilità e specificità di due metodi commerciali adatti alla rilevazione rapida delle ESBL: il terreno chromID ESBL Medium (bioMérieux, Marcy-l'Etoile, France) e il Cica- $\beta$-Test (produttore, Kanto Chemical, Tokio, Japan; distributore europeo, Mast Group, Merseyde, UK).

\section{MATERIALI E METODI}

Lo studio è stato condotto su 121 Enterobatteri raccolti tra Febbraio 2008 e Aprile 2009 presso la sezione di Microbiologia del Laboratorio Analisi dell'IRCCS Istituto Clinico Humanitas.

Sono stati testati un totale di 41 isolati non ripetuti di Escherichia coli, 41 di Proteus mirabilis e 39 di Klebsiella spp..

I ceppi di riferimento E. coli ATCC 25922 e K. pneumoniae ATCC 700603 sono stati inseriti nello studio rispettivamente come controllo negativo e controllo positivo per la produzione di ESBL.

Tutti gli isolati, ottenuti da differenti materiali (urine, sangue, pus, tamponi ferita e campioni provenienti dalle alte/basse vie respiratorie) sono stati inizialmente distinti in ceppi sospetti ESBLproduttori e ceppi non produttori sulla base dei risultati forniti dal sistema Phoenix ${ }^{\mathrm{TM}}$ (BD Diagnostic Systems, Sparks, MD) utilizzato nella pratica diagnostica abituale.

I ceppi sono stati processati utilizzando i pannelli NMIC/ID 55 (BD Diagnostic Systems, Sparks, $\mathrm{MD})$, comprensivi del test per la rilevazione della produzione di ESBL. Questo test è basato sull'utilizzo di pozzetti contenenti cefpodoxime, ceftazidime e ceftriaxone e pozzetti con cefpodoxime, ceftazidime e ceftriaxone più acido calvulanico.

La determinazione dell'avvenuta crescita batterica si ottiene mediante la lettura in continuo delle variazioni nella torbidità batterica all'interno dei pozzetti. I dati di tale lettura vengono elaborati da un particolare algoritmo matematico (Figura I) e controllati da un Sistema Esperto Integrato (ver. 5.75).

La conferma della produzione di ESBL è stata eseguita utilizzando 1'Etest (bioMérieux, Marcyl'Etoile, France).

Tale test fenotipico impiega tre differenti strisce in plastica impregnate ad un'estremità con un gradiente di concentrazione rispettivamente di ceftazidime (TZ) $(0.5-32 \mu \mathrm{g} / \mathrm{mL})$, cefotaxime (CT) e cefepime $(\mathrm{PM})(0.25-16 \mu \mathrm{g} / \mathrm{mL})$, all'altra estremità le strisce presentano la stessa cefalosporina addizionata con clavulanato alla concentrazione di $4 \mu \mathrm{g} / \mathrm{mL}$ : $\mathrm{TZ}+$ clavulanato (TZL) (0.064$4 \mu \mathrm{g} / \mathrm{mL}$ ), CT e PM + clavulanato (CTL e PML) $(0.016-1 \mu \mathrm{g} / \mathrm{mL}+4 \mu \mathrm{g} / \mathrm{mL})$.

Per l'esecuzione di questo test di conferma i ceppi batterici sono stati seminati su Mac Conkey agar (BD Diagnostic Systems, Sparks, MD) ed incubati a $35^{\circ} \mathrm{C}$ per 18 ore.

In seguito è stata preparata, in $5 \mathrm{ml}$ di acqua distillata sterile, una sospensione con torbidità pari a 0.5 McFarland.

Con un tampone sterile è stata eseguita una semina a confluenza della soluzione ottenuta su piastre da $90 \mathrm{~mm}$ di Mueller Hinton agar (bioMérieux, Marcy-l'Etoile, France) su cui sono state poste le strisce (Figura II). 
Dopo incubazione a $35^{\circ} \mathrm{C}$ per 18 ore, i risultati del test sono stati interpretati in accordo ai criteri CLSI 2009 (2).

Primo test in valutazione: le colonie cresciute su agar MacConkey sono state seminate mediante ansa sterile su ChromID ESBL Medium (bioMérieux, Marcy-l'Etoile, France). Il terreno è stato quindi incubato in aerobiosi a $37^{\circ} \mathrm{C}$ per 18 24 ore. Il terreno ChromID ESBL contiene una miscela di antibiotici ed il cefpodoxime, che permettono di inibire selettivamente la crescita di microrganismi non produttori di ESBL.

Tale terreno permette inoltre di definire l'appartenenza alle specie E. coli, Klebsiella spp. e P. mirabilis dei microrganismi cresciuti in piastra grazie al viraggio di colore assunto dalle colonie (Figura III).

Secondo test in valutazione: gli stipiti batterici sono stati seminati sul terreno Mueller Hinton agar (bioMérieux, Marcy-l'Etoile, France).

E stato poi aggiunto un dischetto di cefpodoxime $10 \mu \mathrm{g}$ al fine di provocare un aumento di espressione enzimatica.

Il Cica- $\beta$-Test (produttore, Kanto Chemical, Tokio, Japan; distributore europeo, Mast Group, Merseyde, UK) è stato eseguito dopo un'incubazione di 12 ore a $37^{\circ} \mathrm{C}$.

Il kit contiene come substrato un'ossimino-cefalosporina cromogena, HMRZ-86 che, sensibile all'idrolisi da parte di enzimi di tipo ESBL o AmpC, vira di colore dal giallo al rosso.

Il kit contiene tre tipi di strip che presentano, ad un'estremità, un tampone impregnato rispettivamente di: 1) nessun inibitore delle $\beta$-lattamasi (Test I), 2) acido clavulanico (Test CVA), 3) 2-2benzothienyl acido boronico (Test $\mathrm{C}$ ).

Sulla strip corrispondente al test I è stata dispensata una goccia di substrato (HMRZ-86) equivalente alla concentrazione di $300 \mathrm{mg} / \mathrm{L}$ e sono state aggiunte colonie batteriche di aspetto identico prelevate al margine dell'alone di inibizione.

La lettura del test è stata effettuata dopo 15 minuti: il test è stato considerato positivo in presenza di viraggio di colore (Figura IV) e si è proceduto con il test CVA.

Tale test è stato ritenuto positivo in assenza di viraggio di colore (dovuto alla mancata idrolisi del substrato grazie all'inibizione prodotta dall'acido clavulanico).

Il test $\mathrm{C}$ è stato eseguito in caso di negatività al precedente test. La mancanza del colore rosso è stata ritenuta indice di positività e di presenza di batteri iper-produttori di enzimi di tipo AmpC la cui attività è bloccata dall'acido boronico.

\section{RISULTATI}

L'Etest, quale metodo di riferimento, ha confermato la produzione ESBL in 49/121 stipiti batteri-

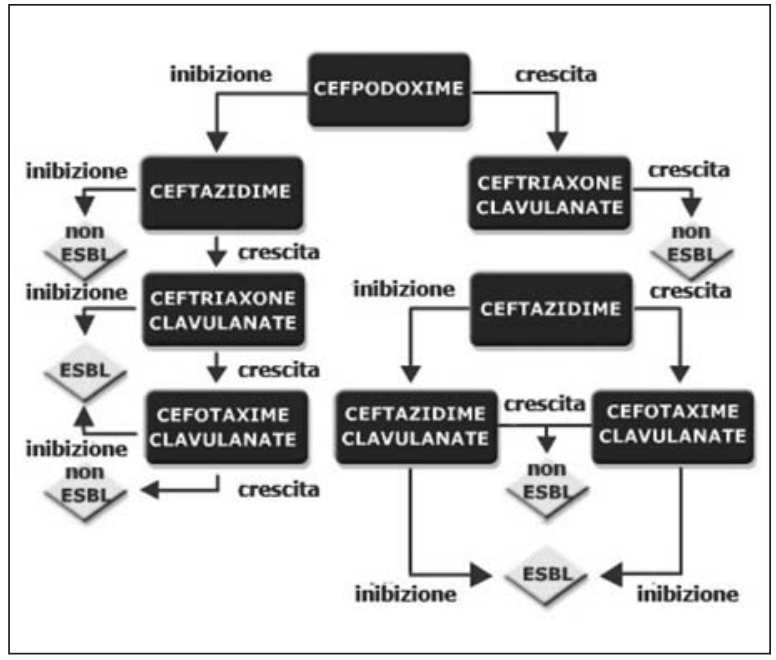

Figura I. Algoritmo matematico di interpretazione del Sistema Esperto ver. 5.75 Phoenix per la rilevazione della presenza di produzione di ESBL.

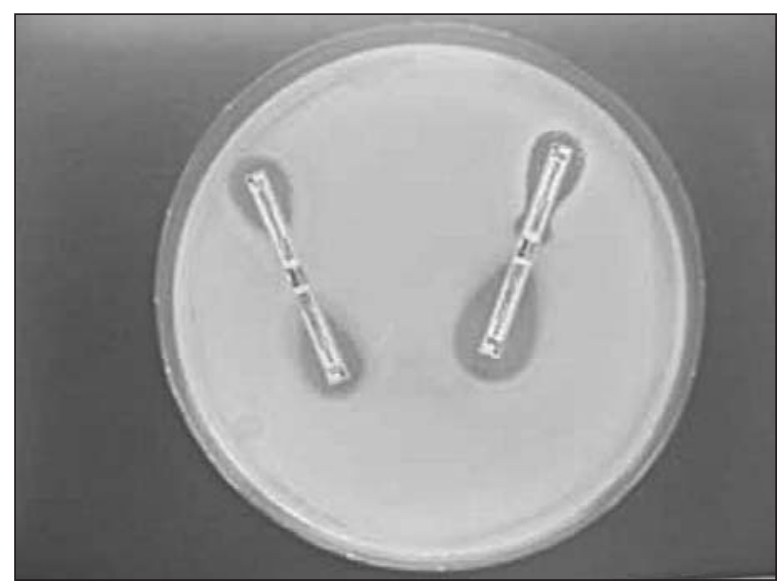

Figura II. Etest: Metodo fenotipico di rivelazione della produzione di enzimi di tipo ESBL.

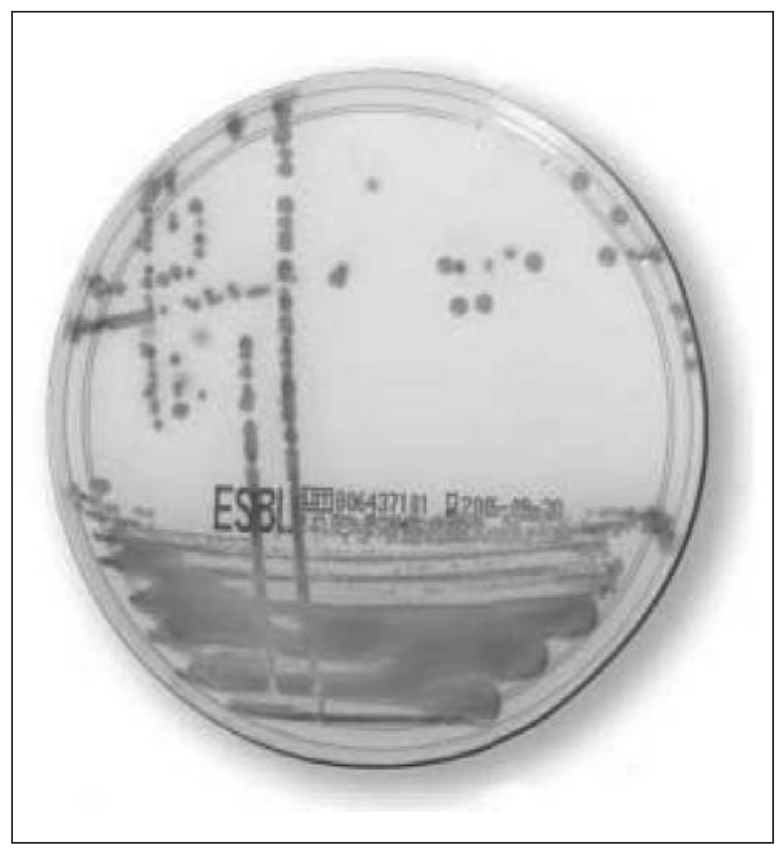

Figura III. Piastra chromID ESBL medium. 
ci precedentemente individuati quali probabili produttori di ESBL dal sistema automatizzato Phoenix.

I 49 Enterobatteri ESBL-produttori comprendevano: 20 stipiti di E. coli, 15 di P. mirabilis e 14 di Klebsiella spp.

Tutti gli isolati batterici sono stati successivamente analizzati con i due test di rilevazione rapida di ESBL in valutazione. I risultati ottenuti sono indicati in Tabella 1.

Etest ha confermato 20/21 isolati di Escherichia coli risultati probabili ESBL-produttori in base all'algoritmo Phoenix ${ }^{\mathrm{TM}}$, le piastre ChromID ESBL Medium 20/21, il Cica- $\beta$-Test 18/21.

Etest ha confermato 15/21 ceppi di Proteus mirabilis risultati probabili ESBL-produttori secondo Phoenix $^{\mathrm{TM}}$; 20/21 di tali ceppi sono risultati positivi mediante le piastre chromID ESBL Medium, mentre $11 / 21$ mediante Cica- $\beta$-Test.

Etest ha individuato come ESBL-produttori 14/19 stipiti di Klebsiella spp. già individuati da Phoenix ${ }^{\mathrm{TM}}$, le piastre chromID ESBL Medium 16/19 e 12/19 Cica- $\beta$-Test.

Sensibilità e specificità dei tre test, dettagliate in Tabella 2.

\section{CONCLUSIONI}

L'emergere sempre più frequente di ceppi produttori di ESBL sia a livello nosocomiale che comunitario rende necessario l'utilizzo routinario, soprattutto nel caso di campioni da infezioni gravi, di test di screening che consentano l'identificazione di ceppi produttori di enzimi idrolizzanti con gravi effetti sull'outcome del paziente.

Nell'ottica dell'introduzione di un test rapido e sensibile per l'identificazione di antibiotico-resistenza nel flusso di lavoro relativo a bacilli Gram negativi isolati da emocolture, sono state valutate sensibilità e specificità di due test commerciali: le piastre cromogene ChromID ESBL ed il Cica- $\beta$-Test.

Farber, et al (4) hanno ottenuto, testando con piastre ChormID 114 ceppi di Enterobacteriaceae,

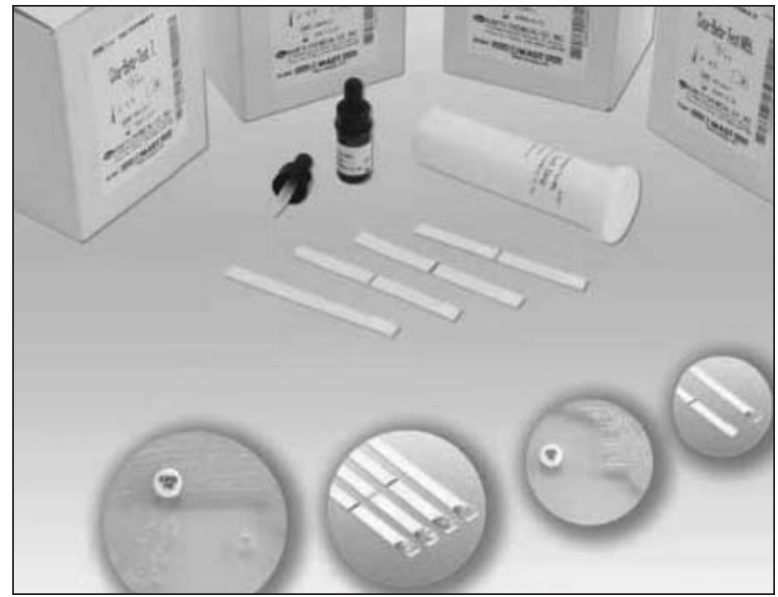

Figura IV. Cica- $\beta$-Test.

ottima sensibilità $(95.8 \%)$ ma una scarsa specificità $(10.5 \%)$ rispetto ad Etest.

Réglier-Poupet $\mathrm{H}$, et al (14), testando 33 ceppi ESBL-produttori già caratterizzati con tecniche molecolari hanno ottenuto una sensibilità pari all' $88 \%$ delle piastre ChormID; anche in questo caso la specificità era bassa e pari al $38.7 \%$. Kasuga, et al (5) utilizzando 47 stipiti batterici, di cui 27 produttori (confermati mediante PCR) e 20 non produttori, hanno raggiunto una sensibilità del $100 \%$, sebbene con una specificità del $20 \%$.

Recentemente Tuchilus, et al (16), testando 112 ceppi di Enterobacteriaceae ed utilizzando come riferimento il gold standard ceftazidime o cefotaxime e la combinazione con clavulanato con Iso-Sensitest agar, hanno ottenuto sensibilità pari al $97 \%$ e specificità pari al $66 \%$.

Il presente studio ricalca in parte i risultati già ottenuti in letteratura (Tabella 1), tuttavia, se si attua una distinzione tra i ceppi di Enterobacteriaceae, si può notare come le performances dei metodi presi in considerazione possano risultare sensibilmente differenti. Nel caso di utilizzo delle piastre di chromiID ESBL medium per stipiti di E. coli, sensibilità e specificità ottenute sono state del $100 \%$. Ciò permette di affermare che tali piastre selettive

Tabella I. Risultati ottenuti dall'analisi dei 12 I ceppi batterici con Phoenix ${ }^{T M}$, chromID ESBL medium, Cica- $\beta$-test ed Etest.

\begin{tabular}{lcccccccc}
\hline & $\begin{array}{c}\text { Phoenix } \\
\text { ESBL }+\end{array}$ & $\begin{array}{c}\text { Phoenix } \\
\text { ESBL }-\end{array}$ & $\begin{array}{c}\text { chromID } \\
\text { ESBL }+\end{array}$ & $\begin{array}{c}\text { chromID } \\
\text { ESBL - }\end{array}$ & $\begin{array}{c}\text { Cica- } \beta \text {-test } \\
\text { ESBL }+\end{array}$ & $\begin{array}{c}\text { Cica- } \beta \text {-test } \\
\text { ESBL - }\end{array}$ & $\begin{array}{c}\text { Etest } \\
\text { ESBL+ }\end{array}$ & $\begin{array}{c}\text { Etest } \\
\text { ESBL- }\end{array}$ \\
\hline E. coli & 21 & 20 & 20 & 21 & 18 & 23 & $\mathbf{2 0}$ & $\mathbf{2 I}$ \\
\hline P. mirabilis & 21 & 20 & 20 & 21 & 11 & 30 & 15 & $\mathbf{2 6}$ \\
\hline Klebsiella spp. & 19 & 20 & 16 & 24 & 12 & 27 & 14 & $\mathbf{2 5}$ \\
\hline
\end{tabular}

Tabella 2. Caratteristiche di sensibilità e specificità espresse da Phoenix ${ }^{T M}$, chromID ESBL medium e Cica- $\beta$-test

\begin{tabular}{lcccccc}
\hline & $\begin{array}{c}\text { Phoenix } \\
\text { Sensibilità }\end{array}$ & $\begin{array}{c}\text { Phoenix } \\
\text { Specificità }\end{array}$ & $\begin{array}{c}\text { chromID } \\
\text { Sensibilità }\end{array}$ & $\begin{array}{c}\text { chromID } \\
\text { Specificità }\end{array}$ & $\begin{array}{c}\text { Cica- } \beta \text {-test } \\
\text { Sensibilità }\end{array}$ & $\begin{array}{c}\text { Cica- } \beta \text {-test } \\
\text { Specificità }\end{array}$ \\
\hline E. coli & $100 \%$ & $95 \%$ & $100 \%$ & $100 \%$ & $90 \%$ & $100 \%$ \\
\hline P. mirabilis & $100 \%$ & $77 \%$ & $100 \%$ & $81 \%$ & $73 \%$ & $100 \%$ \\
\hline Klebsiella spp. & $100 \%$ & $80 \%$ & $100 \%$ & $92 \%$ & $85 \%$ & $100 \%$ \\
\hline
\end{tabular}


cromogene possono essere utilizzate con estrema sicurezza per lo screening delle ESBL per questa specie.

Il Cica- $\beta$-Test era stato precedentemente valutato da Livermore DM, et al (8) sia per la capacità di riconoscere ceppi ESBL-, che AmpC- e metallo$\beta$-lattamasi- produttori. Nello studio sono stati utilizzati sia ceppi di riferimento che ceppi di provenienza clinica: 63/74 ceppi clinici (o transconiugati) produttori di ESBL di classe A, indotti alla produzione degli enzimi mediante semina su Mueller Hinton agar ed apposizione di un dischetto di cefpodoxime, sono stati correttamente riconosciuti ESBL positivi mediante Cica$\beta$-Test.

In base ad uno studio precedente, condotto su 304 stipiti di Klebsiella spp., E. coli e P. mirabilis risultati ESBL-produttori con test del doppio disco, il test presentava una sensibilità pari al $95.5 \%$ ed una specificità del $98.1 \%$ (3).

Uno studio del 2010 condotto con 115 stipiti batterici ESBL- e $67 \mathrm{AmpC}$ - produttori ha evidenziato invece una sensibilità ed una specificità del Cica- $\beta$-Test pari al $94.8 \%$ ed all' $83.8 \%$ rispettivamente (7).

Nel presente studio il Cica- $\beta$-Test ha mostrato una maggiore specificità rispetto al terreno agarizzato cromogeno ChromID, è tuttavia risultato meno sensibile e quindi inadatto quale test di screening relativamente gli scopi prefissati (identificazione microrganismi Gram negativi ESBL-produttori da emocoltra).

Attualmente, presso il Laboratorio Analisi dell'IRCCS Istituto Clinico Humanitas, si procede seminando le emocolture positive per la presenza di bacilli Gram negativi su piastre di Mac Conkey agar, agar sangue di montone e su una piastra di chormID ESBL medium.

In seguito a crescita su chromID ESBL di batteri con colorazione suggestiva di un ceppo di E. coli, il clinico viene avvisato della presenza di un'infezione causata da un probabile ceppo ESBL-produttore.

Questo atteggiamento permette al clinico di correggere la terapia antibiotica empirica impostata con un anticipo di $24-48$ ore rispetto al risultato ottenibile seguendo il flusso routinario (e definitivo).

I dati ottenuti in questo studio mettono in evidenza come, nel caso in cui la batteriemia sia causata da ceppi di Klebsiella spp. o Proteus spp., sia invece necessario proseguire con identificazione biochimica, antibiogramma e con il sistema automatizzato Phoenix, non anticipando il risultato.

Solo nel caso in cui il Sistema Esperto identifica il ceppo come "probabile produttore di ESBL" è opportuno procedere con il test di conferma fenotipico (Etest). Il risultato definitivo potrà giungere al clinico 72 ore dopo l'avvenuta positivizzazione dell'emocoltura.

È pertanto auspicabile che, al più presto, siano messi a punto test genotipici in multiplex PCR o microarray fruibili dai laboratori di microbiologia clinica; ciò, permettano la rivelazione dei più diffusi geni di resistenza, consentirebbe di individuare in tempi stretti isolati problematici dal punto di vista terapeutico ed epidemiologico.

\section{BIBLIOGRAFIA}

1. Bradford PA. Extended-spectrum beta-lactamases in the 21 st century: characterization, epidemiology, and detection of this important resistance threat. Clin Microbiol Rev 2001; 14: 933-51.

2. Clinical and Laboratory Standards Institute 2010. Performance standards for antimicrobial susceptibility testing. Twentieth Informational Supplement. M100-S20.

3. Colodner R, Reznik B, Gal V, et al. Evaluation of a novel kit for the rapid detection of extended spectrum beta-lactamases. Eur J Clin Microbiol Infect Dis 2006; 25: 49-51.

4. Färber J, Moder KA, Layer F, et al. Extended-spectrum Beta-lactamase detection with different panels for automated susceptibility testing and with a chromogenic medium. J Clin Microbiol 2008; 46: 3721-7.

5. Kasuga E, Matsumoto T, Hidaka E, et al. Clinical assessment of novel ChromID ESBL agar plates for detection of ESBL producers in the family Enterobacteriaceae. Rins Bisei Jin Shin Kenku 2009; 20: $1-8$

6. Kollef MH. Inadequate antimicrobial treatment: an important determinant of outcome for hospidalized patients. Clinical Infectious Diseases 2000; 31: 131-8.

7. Lavigne JP, Pfeiffer C, Vidal L, Sotto A. Rapid detection of multidrug resistant Gram-negative bacilli by Cica-Beta-Test strips. Pathol Biol 2010.

8. Livermore DM, Canton R, Gniadkowski M, et al. CTX-M: changing the face of ESBLs in Europe. $J$ Antimicrob Chemother 2007; 59: 165-74.

9. Martinez-Martinez L, Pascual A, Garcia I, et al. Interaction of plasmid and host quinolone resistance. $J$ Antimicrob Chemother 2003; 51: 1037-9.

10. Mohanty S, Gaind R, Ranjan R, Deb M. Use of the cefepime-clavulanate ESBL Etest for detection of extended-spectrum beta-lactamases in AmpC co-producing bacteria. J Infect Dev Ctries 2009; 4: 24-9.

11. M'Zali FH, Chanawong A, Kerr KG, Birkenhead D, Hawkey PM. Detection of extended-spectrum $\hat{a}$-lactamases in members of the family Enterobacteriaceae: comparison of the MAST DD test, the double disk and the Etest ESBL. Antimicrobial Agents and Chemotherapy 2000; 45: 881-5.

12. Pfaller MA, Segreti J. Overview of the epidemiological profile and laboratory detection of extended-spectrum beta-lactamases. Clin Infect Dis 2006 Apr 15; 42 Suppl 4: S153-63.

13. Poirel L, Van De Loo M, Mammeri H, Nordmann P. Association of plasmid-mediated quinolone resistance with extended-spectrum beta-lactamase VEB-1. Antimicrob Agent Chemoter 2005; 49: 3091-4.

14. Réglier-Poupet H, Naas T, Carrer A, et al. Performance of chromID ESBL, a chromogenic medium for detection of Enterobacteriaceae producing extended-spectrum beta-lactamases. J Med Microbiol 2008; 57: 310-5.

15. Sasaki T, Hirai I, Niki M, et al. High prevalence of 
CTX-M $\beta$-lactamase-producing Enterobacteriaceae in stool specimens obtained from healthy individuals in Thailand. J Antimicrob Chemoter 2010; 65: 666-8. 16. Tuchilus C, Poiatã A, Buiuc D. Chromogenic medium for detection of enterobacteria producing beta-lactameses in North-Eastern Romania. Rev Med Chr Soc Med Nat Iasi 2010; 114: 522-5.

17. Tumbarello M, Sali M, Trecarichi EM, et al. Bloodstream infections caused by extended-spectrumbeta-lactamase producing Escherichia coli: risk fac- tors for inadequate initial antimicrobial therapy. Antimicrob Agents Chemother 2008; 52: 3244-52.

18. Yang K, Guglielmo BJ. Diagnosis and treatment of extended-spectrum and AmpC beta-lactamase-producing organisms. Ann Pharmacother 2007; 41: 1427-35.

19. Wu JJ, Ko WC, Tsai SH, Yan JJ. Prevalence of PlasmidMediated Quinolone Resistance Determinants QnrA, QnrB, and QnrS among Clinical Isolates of Enterobacter cloacae in a Taiwanese Hospital. Antimicrob Agent Chemoter 2007; 51: 1223-7. 